\title{
GAMPI
}

\section{Trabalho em equipe e colaboração: oportunidades de pesquisa com estudantes de Design}

Teamwork and collaboration: research opportunities with Design students

SILVA, Arabella N. G.; Doutoranda em Design; UFPR e Unicuritiba

arabella.n.galvao@gmail.com

HEEM AN, Adriano; Doutor; UFPR

adriano.heeman@gmail.com

\section{Resumo}

A habilidade de trabalhar em equipes é exigida do Designer para sua atuação profissional e é exercitada durante sua trajetória acadêmica. Neste contexto, este artigo descreve uma Revisão Bibliográfica Sistemática realizada com o intuito de identificar oportunidades de pesquisa sobre a colaboração em equipes formadas por estudantes de Design. Foram selecionados quinze artigos representativos de áreas do conhecimento distintas, que ofereceram oportunidades de pesquisa. Com base nos resultados, é possível sugerir que as ênfases das pesquisas sobre trabalho em equipe de estudantes se relacionam ao desempenho da equipe, à eficácia do trabalho, à avaliação dos resultados e a aprendizagem do aluno.

Palavras Chave: trabalho em equipe; Design; ensino superior.

\section{Abstract}

The skills for teamwork is required of the Designer for their professional performance and is developed during their academic trajectory. In this context, this paper describes a Systematic Literature Review conducted in order to identify research opportunities for collaboration in teams of Design students. We selected fifteen article representing different knowledge areas and which offered research opportunities. Based on the results, we suggest that the emphasis of the research in student staff relate to the team's performance, the effectiveness of the work, evaluation of results and student learning.

Keywords: teamwork; Design; higher education.

\section{Introdução}

O Design tem se mostrado um campo do conhecimento cada vez mais dinâmico, exigindo de seus profissionais múltiplas capacidades técnicas e comportamentais. Dentre estas capacidades, destaca-se o trabalho em equipe como premissa para facilitar o desenvolvimento de inovações, haja vista a maior possibilidade de geração de ideias em 5o GAM PI Plural, 2015, UNIVILLE, Joinville, SC 


\section{GAMPI
plural ${ }^{45}$}

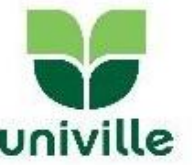

equipes, se comparada ao indivíduo. Kelley (2001) sugere que bons produtos e projetos são, invariavelmente, desenvolvidos por boas equipes e reforça que "o mito do gênio solitário pode, na verdade, atrapalhar os esforços de inovação e criatividade de uma empresa" (KELLEY, 2001, p.90), pois o gênio solitário, normalmente, sente dificuldade em compartilhar suas ideias.

A capacidade de trabalhar em equipes é exercitada pelo Designer desde sua vida acadêmica até a prática profissional. Durante o período da graduação, são inúmeros os trabalhos desenvolvidos pelos futuros Designers em equipes de projeto e, com relativa frequência, os estudantes oferecem resistência a este tipo de trabalho. Montagna et al. (2012) afirmam que os estudantes de Design "preferem normalmente trabalhar individualmente com o objetivo de imprimir nos projetos o seu próprio estilo pessoal". Desta forma, observa-se a oportunidade de um estudo aprofundado sobre as equipes de projetos formadas por estudantes de Design. Além disso, uma pesquisa bibliográfica assistemática mostrou a carência de estudos científicos sobre o trabalho em equipes no campo do Design.

Neste contexto, o objetivo deste artigo é identificar oportunidades de pesquisa sobre a colaboração em equipes de projeto formadas por estudantes de Design. Para atingir o objetivo proposto foi realizada uma Revisão Bibliográfica Sistemática (RBS) sobre o trabalho em equipes de estudantes do ensino superior. Não houve restrição quanto ao campo do conhecimento destes estudantes, pois parte-se da premissa de que métodos, técnicas, ênfases e lacunas identificados em outras áreas poderão evidenciar oportunidades de pesquisa no campo do Design.

A seguir são descritos o Método utilizado, os Resultados alcançados e sua Discussão, bem como a Conclusão do presente trabalho.

\section{Método}

Para identificar oportunidades de pesquisa sobre a colaboração em equipes de projeto formadas por estudantes de Design foi realizada uma Revisão Bibliográfica Sistemática (RBS). A RBS baseia-se na definição de estratégias e métodos sistematizados para realizar buscas de referenciais teóricos e analisar os resultados encontrados, de modo que seja possível repetir o processo por meio de "ciclos contínuos até que os objetivos da revisão sejam alcançados”. (CONFORTO; AMARAL; SILVA, 2011, p. 2).

A RBS que gerou os resultados descritos neste artigo seguiu os seguintes passos: (1) busca de artigos no Portal de Periódicos CAPES; (2) leitura dos títulos dos artigos encontrados; (3) leitura dos resumos dos artigos selecionados a partir dos títulos; (4) leitura da introdução e da conclusão dos artigos selecionados a partir dos resumos; (5) leitura integral dos artigos selecionados a partir da introdução e da conclusão.

A busca de artigos no portal de periódicos da CAPES, primeira etapa desta RBS, foi feita com base nos termos undergraduate e teamwork, na data de 3 de abril de 2015, tendo-se optado pela busca destas palavras exatas em qualquer um dos campos disponíveis (no título, como autor, no assunto). Esta busca encontrou 284 artigos no idioma inglês, no período dos últimos 5 anos.

A segunda etapa desta RBS contemplou a leitura dos títulos dos artigos encontrados na primeira etapa. Foi feita a exclusão de artigos conforme os seguintes critérios: artigos repetidos; artigos que indicassem estudos interprofissionais (por exemplo, estudos de 


\section{GAMPI
plural ${ }^{15}$}

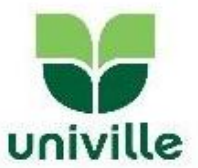

trabalhos em equipe entre enfermeiros e médicos); artigos que tratassem de estudos com profissionais formados. Após estas exclusões, restaram 62 artigos.

Na terceira etapa desta RBS foi feita a leitura dos abstracts dos artigos selecionados na etapa anterior. Esta leitura procurou excluir os artigos segundo os mesmos critérios anteriores, além de privilegiar aqueles que tratavam de colaboração. Nesta etapa foram selecionados 29 artigos.

A leitura da introdução e da conclusão dos artigos selecionados na etapa anterior buscou excluir artigos que descrevessem estudos que não pudessem ser aplicados na área do Design e/ou no Brasil, por diversos motivos. Os motivos mais comuns foram: os estudos utilizaram métodos e/ou ferramentas específicos de uma determinada área do conhecimento e os estudos eram fortemente impactados pelo modelo educacional do país. Além disso, foram ainda excluídos artigos pelos mesmos critérios das etapas anteriores da RBS. Desta forma, restaram 15 artigos para a leitura completa, que serão descritos e discutidos em seguida.

\section{Resultados}

A partir da RBS descrita anteriormente foram selecionados 15 artigos para a leitura completa e identificação de oportunidades de pesquisa sobre a colaboração em equipes de projeto formadas por estudantes de Design. Conforme o Quadro 1, os campos mais recorrentes de aplicação das pesquisas sobre o tema em estudo são o de negócios, com 4 artigos identificados, e o das engenharias e da tecnologia da informação, com 2 artigos identificados em cada um. Os demais artigos são todos de campos do conhecimento diferentes uns dos outros. O Quadro 1 também apresenta uma breve descrição de cada estudo, demonstrando sua diversidade.

\begin{tabular}{|c|c|c|}
\hline REFERÊECIA & DESCRIÇÃO GERAL & $\begin{array}{l}\text { CAMPO DE } \\
\text { APLICAÇÃO }\end{array}$ \\
\hline LIANG, 2012. & $\begin{array}{l}\text { Aplicação de uma ferramenta de apoio ao projeto, para que } \\
\text { estudantes de engenharia trabalhem em equipe na solução de } \\
\text { problemas do sistema de freios do automóvel. }\end{array}$ & $\begin{array}{l}\text { Engenharia } \\
\text { Automotiva }\end{array}$ \\
\hline $\begin{array}{l}\text { PORTER; GOGUS; } \\
\text { YU, } 2010 .\end{array}$ & $\begin{array}{l}\text { Utilização da teoria da alocação de recursos para avaliar o } \\
\text { desempenho de equipes de estudantes de negócios. }\end{array}$ & Negócios \\
\hline $\begin{array}{l}\text { KEMERY; } \\
\text { STICKNEY, } 2014 .\end{array}$ & $\begin{array}{l}\text { Abordagem multifacetada do trabalho em equipe, analisando } \\
\text { desempenho individual e da equipe em provas de } \\
\text { conhecimento do curso. }\end{array}$ & Negócios \\
\hline $\begin{array}{l}\text { RHEE; PARENT; } \\
\text { BASU, } 2013 .\end{array}$ & $\begin{array}{l}\text { Exploração de correlações entre resultados da equipe, } \\
\text { personalidade dos integrantes e habilidade de trabalhar em } \\
\text { equipe. }\end{array}$ & $\begin{array}{l}\text { Eng. Mecânica, Eng. } \\
\text { Elétrica, Design, } \\
\text { Empreendedorismo } \\
\text { Verde e Políticas } \\
\text { Públicas }\end{array}$ \\
\hline $\begin{array}{l}\text { FRENCH; KOTTKE, } \\
2013 .\end{array}$ & $\begin{array}{l}\text { Relações entre a extroversão e a satisfação com o resultado } \\
\text { do trabalho em equipe: hipótese refutada; quanto maior a } \\
\text { extroversão, menor a satisfação com o trabalho. }\end{array}$ & Psicologia \\
\hline $\begin{array}{l}\text { HUGHES; JONES, } \\
2011 .\end{array}$ & $\begin{array}{l}\text { Contribuições dos membros da equipe para a eficácia geral } \\
\text { da equipe, com descrição de alguns instrumentos de } \\
\text { avaliação. }\end{array}$ & $\begin{array}{l}\text { Não cita aplicação } \\
\text { em nenhuma área } \\
\text { específica }\end{array}$ \\
\hline CARVER; & Exame de obstáculos ao trabalho em equipe em cursos de & Direito \\
\hline
\end{tabular}

5o GAM PI Plural, 2015, UNIVILLE, Joinville, SC 


\section{GAMPI
plural ${ }^{45}$}

\begin{tabular}{|c|c|c|}
\hline STICKLEY, 2012. & $\begin{array}{l}\text { graduação em geral e no direito; apresentação método de } \\
\text { implementação do trabalho em equipe com vistas a } \\
\text { minimizar tais obstáculos. }\end{array}$ & \\
\hline RIEBE et al, 2010. & $\begin{array}{l}\text { Ensino do trabalho em equipe como uma competência para a } \\
\text { empregabilidade, apresentado por meio de um estudo de } \\
\text { caso. }\end{array}$ & Negócios \\
\hline $\begin{array}{l}\text { CHEN; HONG; } \\
\text { CHEN, } 2014 .\end{array}$ & $\begin{array}{l}\text { Aplicação de uma ferramenta de orientação ao trabalho em } \\
\text { equipe, de modo colaborativo, para estudantes de TI. }\end{array}$ & $\begin{array}{l}\text { TI - Desenvolvimento } \\
\text { de Software }\end{array}$ \\
\hline $\begin{array}{l}\text { CURRAN; } \\
\text { CARLSON; } \\
\text { CELOTTA, } 2013 .\end{array}$ & $\begin{array}{l}\text { Aplicação de uma ferramenta de aprendizagem colaborativa, } \\
\text { comparando resultados de uma turma que usou a ferramenta } \\
\text { com outra que não usou. }\end{array}$ & Estatística \\
\hline $\begin{array}{l}\text { ALLEN; DONHAM; } \\
\text { BERNHARDT, } \\
2011 .\end{array}$ & $\begin{array}{l}\text { Descrição de evidências da eficácia da aprendizagem } \\
\text { baseada em problemas. }\end{array}$ & Pedagogia \\
\hline GILLESPIE, 2012. & $\begin{array}{l}\text { Aplicação de ferramenta de aprendizagem baseada em } \\
\text { equipe apresentada por meio de estudo de caso. }\end{array}$ & Serviço Social \\
\hline $\begin{array}{l}\text { TSAY; BRADY, } \\
2010 .\end{array}$ & $\begin{array}{l}\text { Estudo que explora a relação entre aprendizagem } \\
\text { colaborativa e desempenho acadêmico no ensino superior. }\end{array}$ & Comunicação \\
\hline $\begin{array}{l}\text { HILLIER; DUNN- } \\
\text { JENSEN, } 2012 .\end{array}$ & $\begin{array}{l}\text { Apresentação de um modelo estruturado, no qual os alunos } \\
\text { aprendem a se tornar uma equipe. }\end{array}$ & Negócios \\
\hline $\begin{array}{l}\text { HIDAYANTO; } \\
\text { SETYADY, } 2014 .\end{array}$ & $\begin{array}{l}\text { Análise do impacto do uso de ferramentas tecnológicas de } \\
\text { colaboração sobre o desempenho acadêmico de alunos. }\end{array}$ & $\begin{array}{l}\text { Ciência da } \\
\text { Computação }\end{array}$ \\
\hline
\end{tabular}

Fonte: os autores, 2015.

\section{Discussão}

Com base nos resultados apresentados anteriormente é possível sugerir que as ênfases das pesquisas sobre trabalho em equipe de estudantes se relacionam ao desempenho da equipe (RHEE; PARENT; BASU, 2013; HUGHES; JONES, 2011; CURRAN; CARLSON; CELOTTA, 2013; TSAY; BRADY, 2010), à eficácia do trabalho (PORTER; GOGUS; YU, 2010; RIEBE et al, 2010), à avaliação dos resultados (KEMERY; STICKNEY, 2014; CARVER; STICKLEY, 2012; HILLIER; DUNN-JENSEN, 2012) e à aprendizagem do aluno (FRENCH; KOTTKE, 2013; CHEN; HONG; CHEN, 2014; GILLESPIE, 2012). Alguns destes estudos ainda destacam dois dos aspectos citados acima, sugerindo serem estas as ênfases mais relevantes para um estudo sobre a colaboração no trabalho em equipes formadas por estudantes de Design. Alguns dos estudos enfatizaram aspectos que não se repetiram, como o uso de ferramentas colaborativas tecnológicas como facilitadoras para o trabalho em equipe (HIDAYANTO; SETYADY, 2014) e o feedback dado aos estudantes em relação ao trabalho em equipe (HILLIER; DUNN-JENSEN, 2012).

Quanto ao método de pesquisa adotado pelos autores estudados, há variações entre os estudos conforme cada abordagem utilizada. No entanto, pode-se afirmar que os instrumentos de coleta de dados privilegiados por estes estudos foram o questionário, para análises quantitativas, e a entrevista, para análises qualitativas.

O estudo realizado por Porter, Gogus e Yu (2010) traz em seu referencial teórico uma distinção entre os conceitos de empreitada e de trabalho em equipe, que podem ser interessantes para a realização de uma pesquisa no campo do Design. Empreitada significa "interações de uma equipe com tarefas, ferramentas e máquinas e inclui esses esforços tradicionalmente associados ao desempenho da tarefa individual" (BOWERS et al., 1997, p. 90 apud PORTER; GOGUS; YU, 2010). Trabalho em equipe refere-se às "interações 5o GAM PI Plural, 2015, UNIVILLE, Joinville, SC 


\section{plural $^{\text {GI }}$}

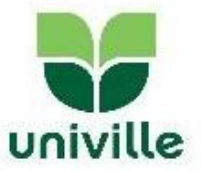

interpessoais entre os membros da equipe que são necessários para a troca de informações, o desenvolvimento e a manutenção de padrões de comunicação e a coordenação" (BOWERS et al., 1997, p. 90 apud PORTER; GOGUS; YU, 2010). Em outras palavras, empreitada é o que as equipes estão fazendo e trabalho em equipe é como elas estão fazendo. O resultado positivo da tarefa depende de um equilíbrio entre os recursos dedicados à empreitada e ao trabalho em equipe (PORTER; GOGUS; YU, 2010). Estas ideias sugerem a possibilidade de realização de uma pesquisa com grupos de estudantes de Design que relacione empreitada, trabalho em equipe e colaboração.

A análise de personalidade dos integrantes de uma equipe foi utilizada como meio para alcançar os objetivos dos estudos por Rhee, Parent e Basu (2013) e French e Kottke (2013). Inclusive, ambos utilizaram o mesmo modelo para identificar as personalidades dentro de um grupo, indicando que este modelo é cientificamente válido. Neste contexto, destaca-se o estudo de Shen et al. (2007 apud RHEE; PARENT; BASU, 2013), que constataram a existência de alguns tipos de personalidade que são melhores para áreas de engenharia e para o campo do Design. Por outro lado, os autores afirmam que uma equipe não deve ser formada por mais de um tipo de personalidade forte em liderança. Estas afirmações oferecem oportunidades de pesquisa sobre o desempenho de equipes de estudantes de Design pelo viés da personalidade de seus integrantes. Contudo, o modelo metodológico precisaria ser complementado para incluir a colaboração entre os integrantes da equipe na análise, em consonância com o objetivo deste artigo.

Também foi sugerido por Shen et al. (2007 apud RHEE; PARENT; BASU, 2013) que, ao formar equipes, não se deve deixar que os alunos as organizem, porque isso reduz a diversidade requerida para ter um grupo de sucesso. Além disso, na descrição dos princípios essenciais da aprendizagem baseada em equipes realizada por Michaelsen \& Knight (2004 apud GILLESPIE, 2012) é fortemente recomendado que a formação do grupo seja realizada pelo instrutor e não pelos estudantes, pelo mesmo motivo já citado. Desta forma, percebe-se outra oportunidade de pesquisa na identificação de como são organizadas equipes de projeto entre estudantes de Design e se o modo como são formadas impacta na colaboração entre seus membros.

Uma constatação importante no estudo conduzido por Michaelsen \& Knight (2004 apud GILLESPIE, 2012) sugere que a tarefa proposta deve ser complexa o suficiente para justificar a abordagem por uma equipe ao invés de um indivíduo, pois desta forma ocorre efetivamente a aprendizagem, tanto de grupo quanto individual. Além disso, Porter, Gogus e $\mathrm{Yu}$ (2010) concluem, após analisar grupos de estudantes de negócios, que quando os estudantes não são confrontados com uma necessidade legítima de trabalho em equipe, em termos de um problema de carga de trabalho, o desempenho do grupo é prejudicado. Esta constatação possui aderência com a forma como agem comumente os educadores do campo do Design, que costumam propor atividades projetuais de maior complexidade para serem realizadas por equipes de alunos. Isso reforça a sugestão de pesquisa sobre o modo como são formados os grupos de trabalho e sua relação com a colaboração.

$\mathrm{Na}$ continuidade das análises, observou-se que Hoegl e Gemuenden (2001 apud CHEN; HONG; CHEN, 2014) desenvolveram um conceito abrangente de colaboração em equipe, denominado TWQ. O TWQ, segundo Chen, Hong e Chen (2014) fornece uma medida prática de colaboração, e sua construção e medidas têm empiricamente sido validadas por outros estudos (CHEN; HONG; CHEN, 2014). Desta forma, sabe-se que já foi definido um 


\section{GAMPIII $_{\text {plural }}$}

método para medir a colaboração em um trabalho em equipe, podendo este método ser aplicado a equipes formadas por estudantes de Design, verificando-se assim outra oportunidade de pesquisa.

Outro estudo que aborda a colaboração foi conduzido por Tsay e Brady (2010). Os pesquisadores realizaram um estudo de caso que teve por objetivo examinar a relação entre a aprendizagem cooperativa e o desempenho acadêmico de alunos num curso superior de comunicação. O estudo foi conduzido na disciplina de metodologia de pesquisa, portanto a tarefa não envolvia uma atividade criativa. Os resultados das análises "sugerem que o envolvimento na aprendizagem cooperativa é um forte preditor do desempenho acadêmico do aluno em sala de aula" (TSAY; BRADY, 2010, p.85). Neste contexto, pode ser identificada uma oportunidade de pesquisa, repetindo-se o mesmo estudo realizado pelos autores, porém no campo do Design.

Os resultados do trabalho em equipe são especialmente avaliados em alguns dos artigos investigados. O Processo de Avaliação de Prontidão (RAP) foi o instrumento de avaliação utilizado por Gillespie (2012) e Tsay e Brady (2010) nos estudos de caso conduzidos por eles. O RAP é composto por um questionário de múltipla escolha (tipicamente 10-20 questões) que atesta a compreensão de conceitos fundamentais dentro de um módulo de aprendizagem, sendo que os alunos realizam a leitura individual de textos introdutórios ao assunto antes da avaliação (GILLESPIE, 2012). Outro instrumento utilizado por vários estudos (KEMERY; STICKNEY, 2014; RHEE; PARENT; BASU, 2013; CARVER; STICKLEY, 2012; RIEBE et al, 2010) e defendido pela literatura de referência sobre o trabalho em equipe é a avaliação por pares, ou seja, quando os próprios alunos avaliam uns aos outros dentro de um grupo. Estes instrumentos, tanto os testes RAP quanto avaliação por pares, poderiam fazer parte de uma pesquisa com grupos de estudantes de Design, como parte do processo de avaliação de desempenho individual e de grupo, da mesma maneira que os autores os utilizaram.

O estudo apresentado por Curran, Carlson e Celotta (2013) apresenta a aplicação da estratégia de aprendizagem colaborativa da maneira como foi concebida inicialmente para o curso de Química e está sendo aplicada em diversos cursos da área de Ciências Exatas. Diversos estudos indicam que a estratégia apresenta resultados positivos para o desempenho dos alunos, porém requer apoio institucional para sua implementação, pois exige remuneração extra para professores e remuneração de estudantes que desempenham o papel de líderes de projeto, já que os estudos dirigidos por esta estratégia configuram-se como atividades extracurriculares. Desta forma, considera-se esta abordagem difícil de ser aplicada como parte de uma pesquisa em cursos de Design, pois se busca uma oportunidade de pesquisa que não exija mudanças em nível institucional, apenas em relação às práticas usadas em sala de aula.

Outra estratégia educacional relevante é a Aprendizagem Baseada em Problemas (PBL - Problem-Based Learning). Allen, Donham e Bernhardt (2011) realizam uma revisão de literatura sobre esta estratégia, exemplificando sua eficácia por meio de diversos estudos. Contudo, os autores destacam que a PBL é particularmente adequada para o desenvolvimento da habilidade de escrita, pois os resultados das atividades de grupo são apresentados sob a forma de uma recomendação, um resumo do que foi aprendido ou alguma outra forma de relatório de grupo ou individual. Não foi relatado nenhum outro tipo de resultado neste estudo, portanto, não é possível saber se esta abordagem seria satisfatória para aplicação em 


\section{GAMPI}

situações cujo resultado seja uma solução criativa materializada sob a forma de um produto, uma peça gráfica, uma coleção de vestuário, etc.

\section{Conclusão}

O Designer deve estar apto a atuar profissionalmente como integrante de uma equipe. Esta competência é exercitada durante sua vida acadêmica. Contudo, o campo do Design carece de pesquisas aprofundadas sobre o trabalho em equipes realizado por alunos do ensino superior. Assim, este artigo buscou identificar oportunidades de pesquisa sobre a colaboração em equipes de projeto de Designers em formação. Para atingir este objetivo foi realizada uma RBS que possibilitou o estudo aprofundado de quinze artigos relevantes sobre o tema.

Os resultados encontrados sugerem que as ênfases das pesquisas sobre trabalho em equipe de estudantes se relacionam ao desempenho da equipe, à eficácia do trabalho, à avaliação dos resultados e à aprendizagem do aluno. Contudo, aspectos teóricos que ainda não foram suficientemente esclarecidos e que, portanto, constituem oportunidades de pesquisa sobre o tema são:

- Relacionamento entre empreitada, trabalho em equipe e colaboração entre os estudantes;

- Identificação de características de personalidade que favoreçam a colaboração entre os estudantes de Design;

- Verificação da maneira como são formadas as equipes de projeto entre estudantes de Design e seu impacto na colaboração entre os membros da equipe;

- Aplicação do método TWQ (HOEGL; GEMUENDEN, 2001 apud CHEN; HONG; CHEN, 2014) para verificar o nível de colaboração em equipes de estudantes de Design;

- Exame da relação entre a aprendizagem cooperativa e o desempenho acadêmico de estudantes de Design.

Este estudo evidenciou também abordagens de difícil aplicação em uma pesquisa com estudantes de Design. Tratam-se de pesquisas que poderiam demandar mudanças institucionais nos cursos nos quais a pesquisa fosse realizada.

Por fim, esta pesquisa descortina possibilidades de estudo sobre o trabalho em equipe e a colaboração entre estudantes de Design. Esclarecimentos a esse respeito poderão contribuir para o desenvolvimento do campo teórico do Design no país.

\section{Agradecimentos:}

Os autores agradecem à Fundação Araucária do Paraná pelo apoio na forma de bolsa de estudos.

\section{Referências}

ALLEN, D. E; DONHAM, R. S.; BERNHARDT, S. A. Problem-Based Learning. New

Directions for Institutional Research, n. 128, p. 21-29, 2011.

CARVER, T. L.; STICKLEY, A. Teamwork in firsty year law units: Can it work? Journal of University Teaching \& Learning Practice, Cambridge, v. 9, n. 2, 2012. 


\section{GAMPI
plural ${ }^{45}$}

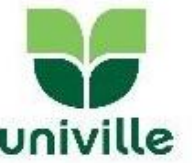

CHEN, C. Y.; HONG, Y. C.; CHEN, P. C. Effects of the meetings-flow approach on quality teamwork in the training of software capstone projects. IEEE Transactions on Education, Taiwan, v. 57, n. 3, p. 201-208, aug. 2014.

CONFORTO, E.C,; AMARAL, D. C.; SILVA, S. L. Roteiro para revisão bibliográfica sistemática: aplicação no desenvolvimento de produtos e gerenciamento de projetos. In: CONGRESSO BRASILEIRO DE GESTÃO DE DESENVOLVIMENTO DE PRODUTO CBGDP, 8., Porto Alegre, 2011. Anais... Porto Alegre: UFRGS, 2011. p. 1-12.

CURRAN, E.; CARLSON, K.; CELOTTA, D. T. Changing attitudes and facilitating understanding in the undergraduate statistics classroom: A collaborative learning approach . Journal of the Scholarship of Teaching and Learning, Indianapolis, v. 13, n. 2, p. 49-71, may. 2013.

FRENCH, K. A.; KOTTKE, J. L. Teamwork satisfaction: Exploring the multilevel interaction of teamwork interest and group extraversion. Active Learning in Higher Education, Thousand Oaks, V. 14, N. 3, P. 189-200, 2013.

GILLESPIE, J. Enhancing social work education through team-based learning. Journal of Social Work Education, Londres, v. 48, n. 2, p. 377-387, 2012.

HIDAYANTO, A. N.; SETYADY, S. T. Impact of collaborative tools utilization on group performance in university students. The Turkish Online Journal of Educational Technology, v. 13, n. 2, p. 88-98, apr. 2014.

HILLIER, J.; DUNN-JENSEN, L. N. Groups Meet . . Teams improve: Building teams that learn. Journal of Management Education, Thousand Oaks, v. 37, n. 5, 704-733, 2012. HUGHES, R. L.; JONES, S. K. Developing and assessing college student teamwork skills. New Directions for Institutional Research, n. 149, p. 53-64, 2011.

KELLEY, Tom. A arte da inovação. Lições de criatividade da IDEO, a maior empresa norte-americana de Design. São Paulo: Futura, 2001.

KEMERY, E. R.; STICKNEY, L. T. A multifaceted approach to teamwork assessment in an undergraduate business program. Journal of Management Education, Thousand Oaks, v. 38, n. 3, p. 462-479, 2014.

LIANG, J. S. Learning in troubleshooting of automotive braking system: a project-based teamwork approach. British Journal of Educational Technology, Oxford, v. 43, n. 2, p. 331-352, 2012.

MONTAGNA, G. et al. O Designer de produto como elemento de ligação nas equipas multidisciplinares. Revista Lusófona de Educação, n.20, 2012, p.99-108. 

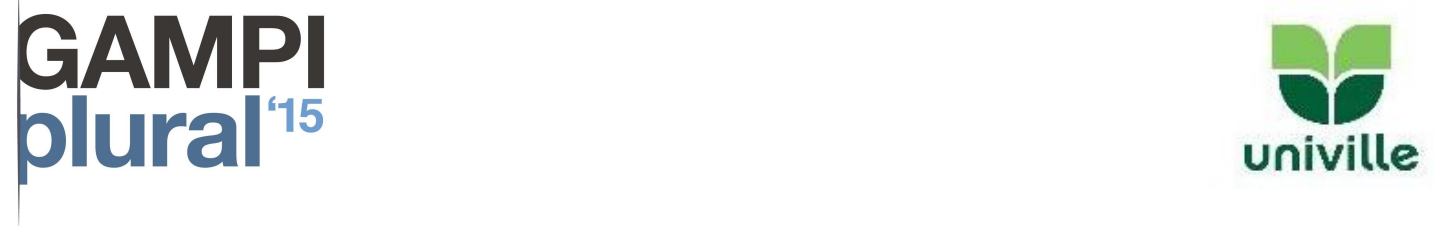

PORTER, C. O. L. H.; GOGUS, C. I.; YU, R. C. F. When does teamwork translate into improved team performance? A resource allocation perspective. Small Group Research, Thousand Oaks, v.41, n. 2, p.221-248, apr. 2010.

RHEE, J.; PARENT, D.; BASU, A. The influence of personality and ability on undergraduate teamwork and team performance. SpringerPlus, Heidelberg, v. 2, n. 1, p. 1-14, 2013.

RIEBE, L. et al. Teamwork: effectively teaching an employability skill. Education + Training, v. 52, n. 6/7, p. 528-539, 2010.

TSAY, M.; BRADY, M. A case study of cooperative learning and communication pedagogy: Does working in teams make a difference? Journal of the Scholarship of Teaching and Learning, Indianapolis, v. 10, n. 2, p. 78-89, jun. 2010. 